\title{
Hilft Traubensilberkerze auch bei Männern gegen Schwitzen durch Hormonentzug?
}

\section{Hintergrund}

Ähnlich Brustkrebspatientinnen unter antihormoneller Behandlung leiden auch viele Männer, die eine antiandrogene Therapie erhalten, an Hitzewallungen und Schwitzen. Extrakten aus Traubensilberkerze (Cimicifuga racemosa) wird bei Frauen Wirksamkeit gegen vasomotorische klimakterische Beschwerden durch östrogenartige Effekte nachgesagt - ist das auf Männer übertragbar? Cimicifuga ist ein Phytoöstrogenräparat, das schon lange zur Linderung von natürlichen oder behandlungsbedingten menopausalen Hitzewallungen eingesetzt wird. Die Wirkung soll durch selektive Östrogenrezeptormodulation vermittelt werden. Sichere Evidenz dafür fehlt jedoch nach wie vor. Die S3-Leitlinie Mammakarzinom [2] stuft Präparate auf der Basis von Traubensilberkerze als „einsetzbar“ ein, konstatiert aber allenfalls geringen Nutzen.

Ein Cochrane-Review bewertet Phytoöstrogenpräparate, u.a. Rotklee und Genistein, als nicht nachgewiesenermaßen wirksam, aber wohl auch nicht als schädlich [3]. Dass sie zu einer Besserung der Symptome führen, wurde in Studien nicht konsistent gezeigt. Auch aktuellere Übersichtsarbeiten fanden keine überzeugenden Belege dafür, dass Traubensilberkerze Wechseljahresbeschwerden lindern kann $[4,5]$.

\section{Keine Daten - keine Empfehlung}

Studien, die die Wirksamkeit von Cimicifuga- oder anderen Phytoöstrogenpräparaten bei Prostatakrebspatienten untersucht haben, sind in medizinischen Literaturdatenbanken nicht dokumentiert. In einer systematischen Übersichtsarbeit [6] formulieren die Autoren ganz allgemein: Verschiedene Studien haben gezeigt, dass Männer, die unter einer Hormonentzugstherapie Hitzewallungen und vergleichbare Beschwerden entwickeln, gut auf die gleichen medikamentösen Therapien ansprechen, die bei Wechseljahresbeschwerden von Frauen wirksam sind. Phytopharmaka sind hier allerdings auch bei Frauen von bisher nicht gesichertem Wert [7].

Unabhängig davon ist ein Behandlungsversuch mit Cimicifuga bei Patienten unter Leuprorelin prinzipiell möglich und birgt kein Risiko. Man muss einem Patienten also nicht unbedingt davon abraten, wenn er gerne „etwas für sich tun“ möchte. Dies kann sich (auch unabhängig von der pharmakologischen Wirkung eines Medikaments) ggf. positiv auf die Lebensqualität auswirken. Es gibt keine Hinweise auf klinisch relevante Wechselwirkungen mit Leuprorelin. Schwerwiegende unerwünschte Wirkungen durch die Einnahme von Cimicifuga wurden bisher nicht beobachtet. Auch sind keine nachteiligen Einflüsse auf den Verlauf einer Prostatakrebserkrankung beschrieben. Im Gegenteil: In vitro- und In-vivo-Daten lassen sogar eine positive Beeinflussung der Erkrankung vermuten [6].

\footnotetext{
Literatur

1. Mucci LA et al (2016) Familial risk and heritability of cancer among twins in Nordic countries. JAMA 315(1):68-76

2. Deutsche Krebsgesellschaft (Hrsg) (2012) Interdisziplinäre S3-Leitlinie für die Diagnostik, Therapie und Nachsorge des Mammakarzinoms. Zuckschwerdt, München
}

3. Lethaby A et al (2013) Phytoestrogens for menopausal vasomotor symptoms. Cochrane Database Syst Rev 2013(12):10

4. Fritz Het al (2013) Black cohosh and breast cancer: A systematic review. Integr Cancer Ther 25: (epub ahead of print)

5. Leach ML, Moore V (2012) Black cohosh (Cimicifuga spp.) for menopausal symptoms. Cochrane Database Syst Rev 12(9):CD007244

6. Drewe J et al (2015) A systematic review of non-hormonal treatments of vasomotor symptoms in climacteric and cancer patients. Springerplus 4:65

7. Adelson KB et al (2005) Treatment of hot flushes in breast and prostate cancer. Expert Opin Pharmacother 6(7):1095-1106

Der Krebsinformationsdienst bietet Informationen zu allen krebsbezogenen Fragestellungen - aktuell, individuell, evidenzbasiert und unabhängig, für die allgemeine Öffentlichkeit und für Fachkreise.

\section{Informationsangebote für Fachkreise}

Telefon: 0800-430 40 50, täglich 8-20 Uhr

E-Mail Service: Krebsinformationsdienst.med@dkfz.de

- Individuelle recherchierte Informationen auf der Basis der besten verfügbaren Evidenz

- Ausführliche Informationen zu mehr als 150 unkonventionellen Diagnose- und Behandlungsverfahren

- Umfassende Datenbank mit Adressen von Krebsberatungsstellen, niedergelassenen Psychoonkologen und weiteren Unterstützungsangeboten

Korrespondenzadresse

A. Gaisser

Krebsinformationsdienst, Deutsches Krebsforschungszentrum andrea.gaisser@dkfz-heidelberg.de

Auszug einer Veröffentlichung aus "Der Onkologe“ 5-2016. 